\title{
Efficient dibutyltin (DBT) elimination by the microscopic fungus Metarhizium robertsii under conditions of intensive aeration and ascorbic acid supplementation
}

\author{
Paulina Siewiera $^{1} \cdot$ Sylwia Różalska $^{1} \cdot$ Przemysław Bernat $^{1}$ \\ Received: 17 November 2016 / Accepted: 7 March 2017 / Published online: 27 March 2017 \\ (C) The Author(s) 2017. This article is published with open access at Springerlink.com
}

\begin{abstract}
Dibutyltin (DBT) is an environmental pollutant characterized by immunotoxic, neurotoxic, and pro-oxidant properties. In this study, an attempt was made to enhance DBT elimination by the Metarhizium robertsii strain. We observed enhanced fungal growth in the bioreactor $\left(\mathrm{pO}_{2} \geq 20 \%\right)$ compared to flask cultures ( $\mu_{\max }$ increased from 0.061 to $\left.0.086 \mathrm{~h}^{-1}\right)$. Moreover, under aerated conditions, $M$. robertsii mycelium with "hairy" morphology biodegraded DBT $\left(20 \mathrm{mg} \mathrm{l}^{-1}\right) 10$-fold faster in the bioreactor than in the flask cultures. Monobutyltin (MBT) and a hydroxylated derivative of MBT $\left(\mathrm{OHBuSnH}_{2}\right)$ were detected as by-products of dibutyltin debutylation. Simultaneous usage of glucose and butyltins indicates the comatabolic nature of monobutyltin and dibutyltin removal. In order to protect fungal cells from oxidative stress caused by DBT presence, vitamin C (20 $\mathrm{mg} \mathrm{l}^{-1}$ ) was applied. Supplementation with ascorbic acid (AA) resulted in a 3 -fold acceleration of MBT removal during the first $7 \mathrm{~h}$ of incubation. Using the HPLC-MS/MS technique, a quantitative analysis of malondialdehyde (MDA), a marker of oxidative stress, was performed. In the AA presence, a decrease in the MDA amount (about 45\%) was observed compared to the case with fungal cells exposed to DBT alone.
\end{abstract}

Responsible editor: Diane Purchase

Electronic supplementary material The online version of this article (doi:10.1007/s11356-017-8764-4) contains supplementary material, which is available to authorized users.

Przemysław Bernat

pbernat@biol.uni.lodz.pl

1 Department of Industrial Microbiology and Biotechnology, Faculty of Biology and Environmental Protection, University of Lodz, Banacha 12/16, 90-237 Lodz, Poland
Keywords Metarhizium robertsii · Dibutyltin utilization · Intense aeration · Hyphae morphology · Antioxidants · Oxidative stress alleviation $\cdot$ Malondialdehyde $\cdot$ Liquid chromatography

\section{Introduction}

Dibutyltin (DBT) is a useful heat stabilizer of polyvinyl chloride, a curing agent for silicone rubbers, and a catalyst for esterification reactions. Because of its widespread use, the compound is found in the environment and in dietary sources (Moser et al. 2009). This organotin is mainly introduced into sediments and water by leaching from PVC materials. Additionally, dibutyltin is formed as a major degradation product of environmental tributyltin (TBT) - the most toxic of all organotin compounds. Due to its hydrophilicity, DBT rapidly enters into water and is accumulated in tissues of mussels and other marine invertebrates (Nesci et al. 2011). The concentrations of DBT in mussels from the Northern Adriatic Sea ranges from 15 to $2660 \mathrm{ng} \mathrm{Sn} \mathrm{g}^{-1}$ (Nemanič et al. 2009). The levels of DBT in mussels collected from the Polish coast (Baltic Sea) are between 0.5 and $24 \mathrm{ng} \mathrm{Sn} \mathrm{g}^{-1}$. On the other hand, dibutyltin found in the liver of European flounder in the Gdansk Bay represents $72-86 \%$ of total butyltin content (Albalat et al. 2002). The presence of DBT in human blood (4.7-36.7 $\mathrm{ng} \mathrm{Sn} \mathrm{ml}^{-1}$ ) and liver samples (0.4-12.8 $\mathrm{ng} \mathrm{Sn} \mathrm{g}^{-1}$ ) has been proved (Whalen et al. 1999; Nielsen and Strand 2002). Liver enzymes are not effective in DBT elimination (Albalat et al. 2002).

In contrast to tributyltin, the toxic effects of dibutyltin are less well known and there is little information available. A reduction of the toxicity of metabolites compared to the initial compound is assumed. Unfortunately, DBT is more immunotoxic to invertebrates and vertebrates than TBT 
(Frouin et al. 2008). Moreover, DBT has a stronger effect on mitochondrial functions than TBT, as it inhibits hydrolytic Mg-ATPase and Ca-ATPase activities in mussels (Bouchard et al. 1999; Nesci et al. 2011). A potential neurotoxic effect of DBT, which may lead to apoptotic death of the hippocampus and neocortex cells in rats, has also been reported (Jenkins et al. 2004). DBT is an inducer of oxidative stress and an amplifier of pro-inflammatory cytokine expression in microglia cells (Chantong et al. 2014).

In this study, an attempt was undertaken to improve the process of dibutyltin biodegradation using a microscopic fungus Metarhizium robertsii. The selected strain can eliminate TBT with high levels of efficiency by protecting the fungal cells from oxidative stress through the application of $17 \beta$-estradiol (Siewiera et al. 2015). In the present research, ascorbic acid (AA, vitamin $\mathrm{C}$ ) and $\alpha$-tocopherol (vitamin $\mathrm{E}$ ) were chosen as primary antioxidants of the aqueous and lipophilic phases, respectively (Li and Schellhorn 2007). The effectiveness of vitamins in free radical scavenging was verified by quantitative analysis of malondialdehyde (MDA), a lipid peroxidation product. In order to speed up the process of DBT degradation, an additional oxygen supply $\left(\mathrm{pO}_{2} \geq 20 \%\right)$ was prepared. Moreover, there was an attempt at the identification of metabolic intermediates formed during organotin dealkylation.

\section{Materials and methods}

\section{Chemicals}

Dibutyltin dichloride, ascorbic acid, $\alpha$-tocopherol, methyl magnesium bromide, tropolone, anhydrous sodium sulfate, and 1,1,3,3-tetraethoxypropane were purchased from SigmaAldrich Chemical Co. (Germany). Stock solutions of $\mathrm{DBTCl}_{2}, \alpha$-tocopherol (vitamin E), and ascorbic acid (vitamin C), each at a concentration of $10 \mathrm{mg} \mathrm{ml}^{-1}$, were prepared in ethanol, dimethyl sulfoxide, and distilled water, respectively. The solvents for organotin extraction such as methanol, hexane, and ethyl acetate were purchased from POCH S.A. (Poland). Other high purity organic solvents used during gas and liquid chromatography analyses originated from J.T. Baker Chemical Co. (the Netherlands).

\section{Microorganism and growth conditions}

The ascomycete insect pathogenic fungus $M$. robertsii IM 6519 from the Department of Industrial Microbiology and Biotechnology (University of Lodz, Poland) was the subject of the study. The ability of the microorganism to degrade organotins was confirmed in an earlier paper (Siewiera et al. 2015).

Fourteen-day-old fungal cultures on ZT slants were used to inoculate synthetic medium (Lobos et al. 1992) in 100-ml Erlenmeyer flasks. The medium was modified and consisted of (grams per liter) $\mathrm{K}_{2} \mathrm{HPO}_{4}$ (4.36), $\mathrm{KH}_{2} \mathrm{PO}_{4}$ (1.7), $\mathrm{MgSO}_{4}$. $7 \mathrm{H}_{2} \mathrm{O}(0.2), \mathrm{MnSO}_{4}(0.05), \mathrm{FeSO}_{4} \cdot 7 \mathrm{H}_{2} \mathrm{O}(0.01), \mathrm{CaCl}_{2} \cdot 2 \mathrm{H}_{2} \mathrm{O}$ (0.03), glucose (40), yeast extract (10), and distilled water (up to 1 1), $\mathrm{pH} 6.8$. The cultivation was carried out at $28^{\circ} \mathrm{C}$ with shaking at $160 \mathrm{rpm}$ for $24 \mathrm{~h}$. The precultures were transferred to fresh medium (1:1 ratio) and incubated for another $24 \mathrm{~h}$. In 100 -ml flasks, the synthetic medium with DBT $\left(20 \mathrm{mg}^{-1}\right)$ or without the organotin (the control cultures) was inoculated with $20 \%$ of a homogeneous preculture. Incubation was conducted for $120 \mathrm{~h}$ in the above--mentioned conditions. Samples for analyses were collected after $0,24,48,72,96$, and 120-h cultivations.

\section{Batch cultivations}

Batch cultivations of the $M$. robertsii strain were conducted in a 3.6-1 bioreactor (Labfors 5; Iris 6 software; Infors AG, Switzerland) with a culture volume of 11 . The fungal preculture, obtained as described above, was additionally transferred to the fresh medium (1:2 ratio) and incubated for a further $24 \mathrm{~h}$. Finally, the homogeneous preculture was introduced into $800 \mathrm{ml}$ of the synthetic medium with DBT (20 $\mathrm{mg} \mathrm{l}^{-1}$ ), either alone or in a mixture with one of the vitamins (C or E, both $20 \mathrm{mg} \mathrm{l}^{-1}$ ) or without the tested compounds (the control culture). The applied concentrations of the vitamins did not affect $M$. robertsii growth. The fungal cultures were incubated for $72 \mathrm{~h}$ with controlled aeration (air flow $\left.11 \mathrm{~min}^{-1}\right)$, stirring $(200-250 \mathrm{rpm})$, temperature $\left(28^{\circ} \mathrm{C}\right)$, and level of dissolved oxygen $\left(\mathrm{pO}_{2} \geq 20 \%\right)$. The quantities of $\mathrm{O}_{2}$ in the introduced and exhaust gasses were measured with a gas analyzer (Infors AG, Switzerland). The $\mathrm{pH}$ of the medium was not regulated during the cultivation. In order to determine the fungal growth and DBT utilization, the samples were collected regularly: after $0,3,7,12,24,48$, and $72 \mathrm{~h}$.

\section{Fungal biomass estimation}

Fungal mycelia were separated from culture media by filtration through Whatman\#1 filter paper and drying at $105{ }^{\circ} \mathrm{C}$ to reach a constant weight. The maximum specific growth rate $\left(\mu_{\max }\right)$ was calculated in accordance with the formula $\mu_{\max }=\left[\left(\ln X_{2}-\ln X_{1}\right) /\left(t_{2}-t_{1}\right)\right]$, where $X_{2}$ is the biomass concentration at time $t_{2}$ and analogously for $X_{1}$. Based on the growth curves, logarithmic growth phases of the $M$. robertsii strain were indicated. Mycelium amounts required for the calculations were taken from 0 and 24-h incubation and from 3 and 7-h cultivation for flask and batch cultures, respectively.

\section{Glucose content analysis}

Glucose amounts in the supernatant were determined using an Agilent 1200 HPLC coupled with a QTRAP 3200 mass 
spectrometer (AB Sciex), according to the Bernat et al. (2013) procedure.

\section{Sample preparation and organotin determination}

Acidified ( $\mathrm{pH}$ 2) fungal biomass was suspended in methanol and homogenized with glass beads by ball milling (Retsch MM 400, Germany). After disruption, the samples were prepared according to the procedure by Bernat et al. (2013).

\section{The analysis of butyltin intermediates}

The cultures $(20 \mathrm{ml})$ were transferred into Falcon tubes and centrifuged for $10 \mathrm{~min}$ at $10,000 \times \mathrm{g}$. The mycelium was suspended in methanol and homogenized using a mixer mill with glass beads for $5 \mathrm{~min}$ at $30 \mathrm{~m} \mathrm{~s}^{-1}$. The extraction of the homogenate and supernatant mixture with ethyl acetate $(1: 1$ $v / v$ ) was carried out twice. The organic layers were dehydrated with the use of anhydrous sodium sulfate and evaporated to dryness. The precipitate was dissolved in methanol.

Separation of butyltins was performed with the Agilent Technologies 1200 HPLC system equipped with a Phenomenex Aqua C18 $125 \mathrm{~A}$ column $(50 \mathrm{~mm} \times 2.0 \mathrm{~mm} \times 5 \mu \mathrm{m})$ and maintained at $37^{\circ} \mathrm{C}$. The mobile phase consisted of water (A) and methanol (B), both supplemented with $2 \mathrm{mM}$ ammonium formate and $0.2 \%$ formic acid. The run time was $9 \mathrm{~min}$, and the solvent gradient was initiated at $60 \% \mathrm{~B}$. After $1 \mathrm{~min}$, the amount of B was increased to $100 \%$ over the following $2 \mathrm{~min}$, and this was maintained for two additional minutes before returning to the initial solvent composition over the next $2 \mathrm{~min}$, and this then being maintained for $2 \mathrm{~min}$. The flow rate was $0.5 \mathrm{ml} \mathrm{min}^{-1}$ with an autosampler temperature of $10^{\circ} \mathrm{C}$ and an injection volume of $10 \mu \mathrm{l}$, respectively.

To identify DBT derivatives in fungal samples by LC-MS/ $\mathrm{MS}$, an information-dependent acquisition (IDA) method was developed consisting of a precursor ion scan (PI) and an enhanced product ion (EPI) scan mode. IDA experiments were performed on a hybrid Q-Trap 3200 mass spectrometer (QTRAP; AB Sciex) connected to the HPLC system. The ion source conditions were set as follows: curtain gas $(\mathrm{CUR})=25$, collision gas $(\mathrm{CAD})=$ high, ionspray voltage $(\mathrm{IS})=5500$, temperature $(\mathrm{TEM})=500$, ion source gas 1 $(\mathrm{GS} 1)=40$, and ion source gas $2(\mathrm{GS} 2)=50$. Nitrogen was used as a nebulizer and an auxiliary gas. For the PI-EPI analysis, a PI scan of $m / z 179\left(\mathrm{BuSnH}_{2}{ }^{+}\right)$was run in positive mode at a scan range from $m / z, 200$ to $m / z, 650$. The EPI scan was run in positive mode at a scan range for daughter ions from $\mathrm{m} / \mathrm{z}$ 100 to $m / z$ 700. Declustering potential (DP), entrance potential (EP), and collision energy (CE) were set to 25,10 , and 28, respectively.

\section{Characterization of morphological modifications}

In order to determine the morphology of the $M$. robertsii hyphae from the exponential growth phase, image analysis was used. Fungal pellet morphology was performed for 30 pellets from each culture using the software package Axiovision 4.4 (Carl Zeiss, Germany). According to the Casas López et al. (2005) method, a central compact core region and a peripheral "hairy" region of the fungal pellets were separated. Subsequently, values of the pellet and pellet core projected area $\left(\mathrm{mm}^{2}\right)$ were estimated.

\section{Quantitative determination of malondialdehyde}

Samples were prepared according to the procedure by Wei et al. (2010) with some modifications. After $7 \mathrm{~h}$ of batch cultivation, $10 \mathrm{ml}$ of the culture was collected and filtrated. The mycelium was washed with distilled water and transferred into Eppendorf tubes containing $1 \mathrm{ml}$ of cooled water and glass beads. The homogenization process with the use of FastPrep 24 (MP Biomedicals, USA) was performed three times for $20 \mathrm{~s}$ at $4 \mathrm{~m} \mathrm{~s}^{-1}$ with 2-min breaks for cooling samples on ice. Subsequently, samples were centrifuged without beads for $10 \mathrm{~min}$ at $4000 \times \mathrm{g}$ at $4{ }^{\circ} \mathrm{C}$. The upper layers were transferred into inserts of dark glass vials for LC-MS/MS analysis. According to the Csallany et al. (1984) procedure, the MDA standard for quantitative determinations was obtained by acid hydrolysis of 1,1,3,3-tetraethoxypropane.

Measurement of MDA was performed using an Agilent 1200 HPLC (Santa Clara CA, USA) system and a 3200 QTRAP mass spectrometer (AB Sciex, Framingham, MA, USA) with an ESI source. For reversed-phase chromatographic analysis, $10 \mu \mathrm{l}$ of the sample was injected into a Phenomenex Aqua C18 125A column $(50 \mathrm{~mm} \times 2.0 \mathrm{~mm} \times 5 \mu \mathrm{m})$. The mobile phase consisted of water (A) and methanol (B); $5 \mathrm{mM}$ ammonium formate was also used in all solvents as an additive. The solvent gradient was initiated at $20 \% \mathrm{~B}$; after $0.5 \mathrm{~min}$, this was increased to $100 \% \mathrm{~B}$ over $1 \mathrm{~min}$ and maintained at $100 \% \mathrm{~B}$ for four additional minutes before returning to the initial solvent composition over $2 \mathrm{~min}$. The run time was $7 \mathrm{~min}$. The column temperature was maintained at $37{ }^{\circ} \mathrm{C}$, and the flow rate was $600 \mu \mathrm{l} \mathrm{min}{ }^{-1}$. The instrumental settings were as follows: spray voltage $-4500 \mathrm{~V}$, curtain gas (CUR) 25, nebulizer gas (GS1) 55, turbo gas (GS2) 60, and ion source temperature of $500{ }^{\circ} \mathrm{C}$. Data analysis was performed with Analyst ${ }^{\mathrm{TM}}$ v1.5.2 software (AB Sciex, Framingham, MA, USA). The monitored multiple reaction monitoring (MRM) pair for MDA was $m / z$ 71-42.

\section{Statistical analysis}

The experiments were carried out with triplicate samples. The Student's $t$ test and Spearman's correlation were performed 
using Excel 2007 (Microsoft Corporation, USA). An average standard deviation $( \pm \mathrm{SD})$ was calculated. Values were considered significant when $p \leq 0.05$.

\section{Results and discussion}

\section{Growth kinetics and DBT biotransformation by the $M$. robertsii strain incubated in flasks}

Based on the results of preliminary studies (data not shown), synthetic medium with an addition of yeast extract as a source of both organic nitrogen and various vitamins (Lee and Little 2015) was chosen for the M. robertsii strain cultivation. The growth kinetics and glucose assimilation by the fungal cells are shown in Fig. 1a. After 120-h incubation, the biomass amount was almost $16 \mathrm{~g} \mathrm{l}^{-1}$, while the glucose uptake was complete after $72 \mathrm{~h}$ of cultivation. In the presence of DBT $\left(20 \mathrm{mg}^{-1}\right)$, an increase in the fungal dry weight of about $22 \%$ was observed (Fig. 1b). During the first $24 \mathrm{~h}$ of the experiment, glucose assimilation by those fungal cells exposed to the organotins was lower than in control cultures. At the same time, the most rapid decrease in DBT level was observed (Fig. 1b). No significant differences were noted in the substrate assimilation after $72 \mathrm{~h}$ of cultivation between the cultures, either with or without DBT. Due to the simultaneous utilization of glucose and the organotins, the cometabolic
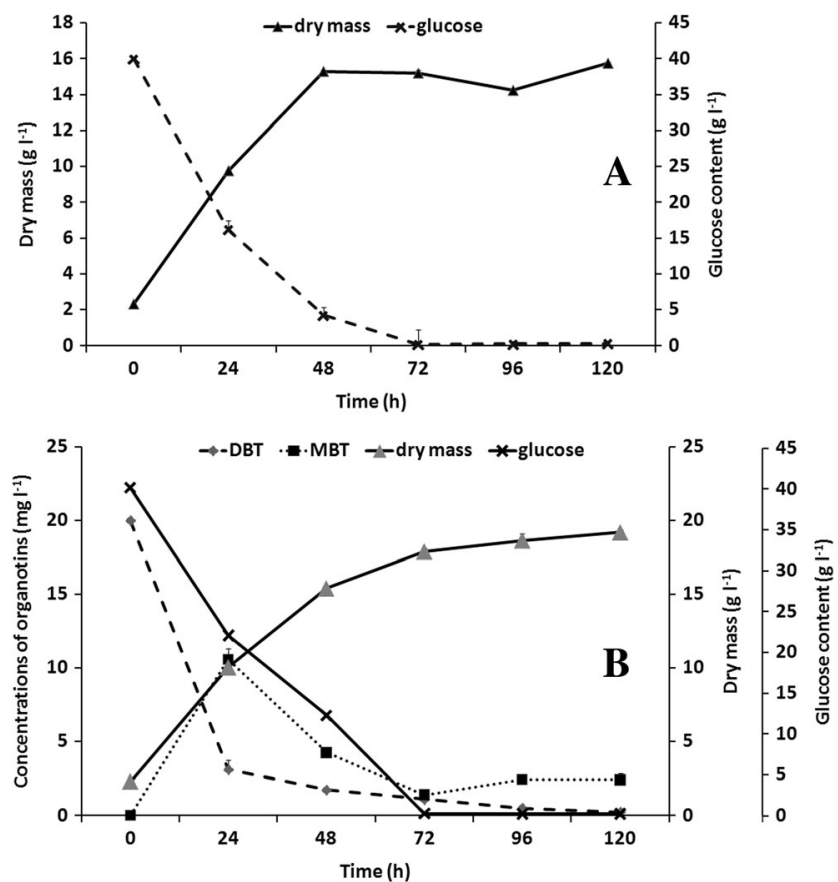

Fig. 1 Biomass synthesis and glucose assimilation by the M. robertsii strain during flask cultivation without (a) and with an addition of DBT (b) for $120 \mathrm{~h}$ in synthetic medium. Additionally, in $\mathbf{b}$, the curves for DBT and MBT biodegradation by the examined fungus incubated in the above conditions are shown character of the compound removal was indicated. The same phenomenon has previously been described for DBT biodegradation by Cochliobolus lunatus and TBT elimination by both Cunninghamella elegans and M. robertsii (Bernat and Długoński 2006; Bernat et al. 2013; Siewiera et al. 2015).

The ability of the M. robertsii strain to eliminate not only dibutyltin but also its derivative compound-monobutyltin (MBT) - was confirmed. The efficiency of DBT removal after 5-day incubation on modified synthetic medium was estimated as $98 \%$. On the other hand, a fifth of the MBT remained in the culture (Fig. 1b).

\section{Fungal growth and organotin utilization}

by the $M$. robertsii strain cultivated under intensive aeration conditions

Although hyphae are not as sensitive as tissues (Bilodeau et al. 2005), mechanical agitation in a bioreactor undoubtedly causes stress resulting in morphological modifications in microorganism cells (Boswell et al. 2003; Chamsartra et al. 2005). However, the benefits of bioprocesses carried out on a bioreactor scale, such as culture homogeneity and facilitated transfers of nutrients, respiration gasses, and metabolic products (Garcia-Ochoa and Gomez 2009), seem to be more important than damage caused by mechanical stress. One positive impact of intensive oxygenation on fungal growth and organotin biodegradation has previously been discovered (Bernat and Długoński 2006). Therefore, in the next stage of the study, an experiment was performed with the aid of a bioreactor. After $72 \mathrm{~h}$ of batch cultivation, the fungal biomass from the control culture was lower (about $30 \%$ in comparison with the flask cultures) (Fig. 2a). Maximum specific growth rates $\left(\mu_{\max }\right)$ of the $M$. robertsii cells were also determined. The parameters for the fungus cultivated without DBT both in flask and batch cultures were 0.060 and $0.078 \mathrm{~h}^{-1}$, respectively. The acceleration of fungal growth in the bioreactor was caused by the efficient system supply of $\mathrm{O}_{2}$. Oxygen plays a key role in aerobic processes, especially in microorganism growth and metabolite production (Garcia-Ochoa and Gomez 2009). In our studies, the minimal level of dissolved oxygen was $20 \%$. In the presence of DBT, $\mathrm{pO}_{2}=20 \%$ was achieved after $17 \mathrm{~h}$ of incubation, $5 \mathrm{~h}$ later than in the fungal culture without the organotin. The changes in the productivity of the fungal biomass exposed to DBT were not significant (Fig. 2b), despite an increase in the $\mu_{\max }$ value up to $0.086 \mathrm{~h}^{-1}$. A reverse dependency was observed in flask cultures supplemented with DBT. The maximum specific growth rates of the $M$. robertsii cells remained constant, despite the increase in the biomass amount compared to the control culture.

The rate of butyltin biotransformation by the fungal strain cultivated under intensive aeration conditions is presented in Fig. 2b. Although the final effectiveness of DBT elimination is comparable to the rate of the compound removal in flask 

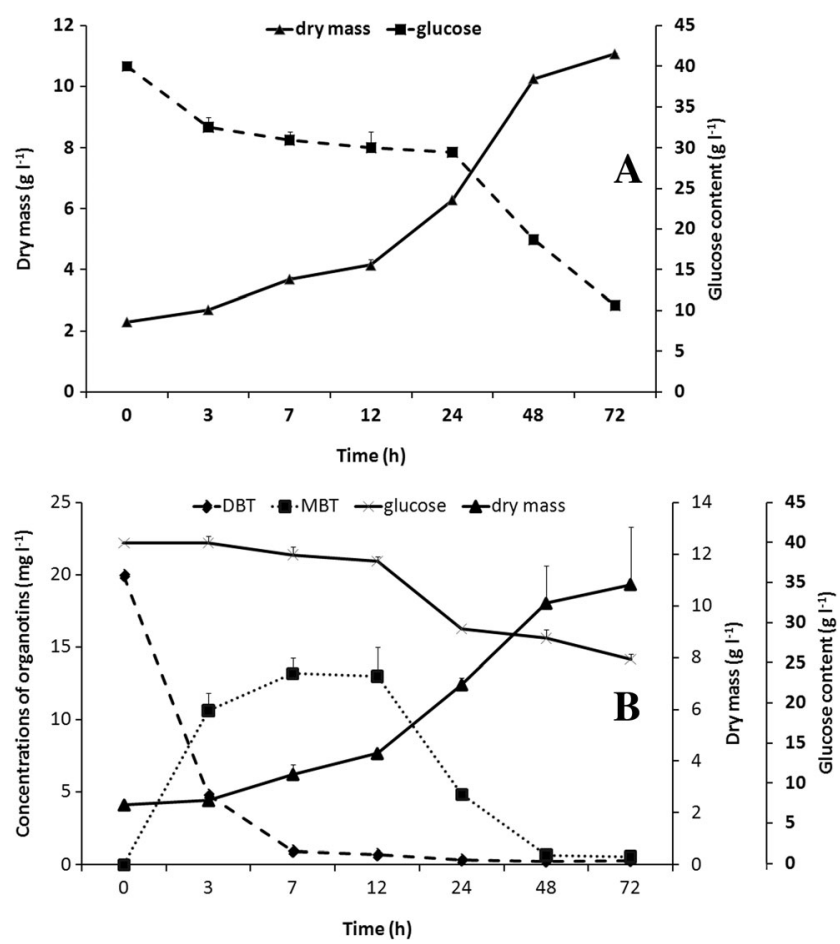

Fig. 2 Biomass synthesis and glucose assimilation by the M. robertsii strain during batch cultivation without (a) and with an addition of DBT (b) for $72 \mathrm{~h}$ in synthetic medium. Additionally, in $\mathbf{b}$, the curves for DBT and MBT biodegradation by the examined fungus incubated in the above conditions are shown

cultures, a supply of oxygen resulted in a 10-fold acceleration of dibutyltin removal. A similar effect was described by Bernat and Długoński (2006) for TBT utilization by an additional oxygen supply to a growing C. elegans strain. The influence of the level of culture oxygenation on the progress of DBT debutylation by the $M$. robertsii is crucial. Moreover, complete metabolism of MBT by the fungus was possible only in the bioreactor culture. The positive effect of batch conditions on fungal growth and the organotin utilization by the microorganism is connected with a facilitated exchange of nutrients and respiratory gasses between the biomass and growth medium (Garcia-Ochoa and Gomez 2009).

In contrast to TBT, a compound biodegraded by bacteria (e.g., Moraxella osloensis (Yáñez et al. 2015), Pseudomonas sp. (Bernat et al. 2014), and Enterobacter cloacae (Sakultantimetha et al. 2011)), fungi (e.g., C. elegans (Bernat and Długoński 2006), Cunninghamella echinulata (Soboń et al. 2016), and Coniophora puteana (White et al. 1999)), the alga Chlorella vulgaris (Tsang et al. 1999), and crab Thalamita crenata (Chen et al. 2016), DBT and MBT have been described as being degraded by only a few microbial strains. Among the fungi, only $C$. lunatus has been mentioned as a strain efficiently degrading dibutyltin and monobutyltin. During incubation on Sabouraud medium, 92\% of the initial DBT concentration $\left(10 \mathrm{mg} \mathrm{l}^{-1}\right)$ and approximately $70 \%$ of formed MBT were removed from the fungal culture after 24 and $168 \mathrm{~h}$ of cultivation, respectively (Bernat et al. 2013). On the other hand, the yield of DBT (20 $\left.\mathrm{mg} \mathrm{l}^{-1}\right)$ transformation by Streptomyces sp. was equal to $90 \%$ after 1day cultivation on synthetic medium with a 2-fold higher amount of MBT in comparison to the $C$. lunatus strain. Moreover, within 7 days, almost $90 \%$ of produced MBT was removed from the bacterial culture (Bernat and Długoński 2009). The most significant advantage of our results, compared to those obtained in other published studies, is the reduction of the time required for the bioremediation process. During the first $24 \mathrm{~h}$ of cultivation, differences were slight, because all strains achieved a high (90\%) efficiency of DBT elimination. However, MBT was still detected as a major byproduct (until day 7), in both Streptomyces sp. (Bernat and Długoński 2009) and C. lunatus (Bernat et al. 2013) cultures, while as early as after 2 days of the M. robertsii cultivation, both butyltins were completely eliminated.

Unfortunately, no literature data concerning the comparison of dibutyltin elimination efficiency in flask and batch conditions are available. According to Moscoso et al. (2012), benzo[a]anthracene (BaA), one of the polycyclic aromatic hydrocarbons, is biodegraded by Staphylococcus warneri and Bacillus pumilus strains more efficiently in batch experiments than in flask conditions. After 2-day cultivation of the bacterial consortium on minimal medium with $\mathrm{BaA}$ $(100 \mu \mathrm{M}), 8$ and $75 \%$ of initial compound concentration were removed in flask and bioreactor, respectively (Moscoso et al. 2012). On the other hand, in further studies on BaA biotransformation in the same conditions by the bacterial strain Pseudomonas stutzeri, a reverse dependency was observed. The efficiency of BaA utilization was equal to $94 \%$ in flasks and $81 \%$ in the bioreactor after 7 days of bacterial incubation (Moscoso et al. 2015). The published results indicate the importance of the metabolic abilities of microorganisms.

\section{The influence of vitamins $C$ and $E$ on DBT removal by the examined fungus}

In our previous paper (Siewiera et al. 2015), an increase in the efficiency (about 14\%) of TBT elimination by the $M$. robertsii strain cultivated in the presence of $17 \beta$-estradiol was confirmed. DBT, as well as TBT, promotes production of reactive oxygen species (ROS) (Chantong et al. 2014), which can inflict direct damage on cell components. In order to increase fungal tolerance to oxidative stress induced by the presence of DBT, vitamins $\mathrm{C}$ and $\mathrm{E}$ were applied. The influence of ascorbic acid $\left(20 \mathrm{mg} \mathrm{l}^{-1}\right)$ or $\alpha$-tocopherol $\left(20 \mathrm{mg} \mathrm{l}^{-1}\right)$ on fungal biomass synthesis and organotin biodegradation is demonstrated in Fig. 3. In the presence of vitamins, the assimilation of glucose was about 50\% higher for vitamin C (Fig. 3a) and about $68 \%$ higher for vitamin $\mathrm{E}$ (Fig. 3b) compared to culture with DBT alone. Moreover, biomass production increased about 34 and $58 \%$, respectively. After supplementation of 
the growth medium with the tested vitamins, the values of $\mu_{\max }$ were decreased compared to the control cultures and remained at the same level $\left(\sim 0.063 \mathrm{~h}^{-1}\right)$. In the presence of antioxidants, faster oxygen uptake from growth medium by fungal cells was observed in comparison to the culture with DBT alone. $\mathrm{pO}_{2}=20 \%$ was achieved after 14 and $9 \mathrm{~h}$ for vitamins $\mathrm{C}$ and $\mathrm{E}$, respectively.

Vitamin supplementation played a greater role in MBT than in DBT elimination. The addition of ascorbic acid to the fungal culture led to a 3 -fold acceleration of the rate of monobutyltin decomposition. The importance of vitamin $\mathrm{C}$ in heavy metal removal by the filamentous fungi was noted. According to Słaba et al. (2013), the addition of $1 \mathrm{mM}$ ascorbic acid enhanced the uptake of lead and copper into the cell walls of Paecilomyces marquandi. In this study, in contrast to vitamin $\mathrm{C}$, the negative impact of $\alpha$-tocopherol on the MBT elimination was observed (about $40 \%$ during the first $7 \mathrm{~h}$ of incubation). The involvement of cytochrome P450 enzymes in vitamin E metabolism has been proved (Sontag and Parker 2002). Thus potentially, $\alpha$-tocopherol could be a competitive inhibitor of CYP450, decreasing the rate of organotin debutylation. However, the involvement of the enzymatic complex in butyltin biodegradation by the M. robertsii strain has not yet been examined. On the other hand, the importance of vitamin $\mathrm{E}$ was confirmed in the biodegradation of polychlorobiphenyls as environmental pollutants. According to Ponce et al. (2011), $\alpha$-tocopherol $(1.6 \mu \mathrm{M})$ improved degradation of biphenyl and 4-chlorobiphenyl (4-CB) by the bacterial strain Burkholderia xenovorans cultivated in an aqueous solution. In the presence of the antioxidant, an increase in the rate of biphenyl degradation $(40 \mu \mathrm{M})$ from $0.8 \pm 0.1$ to $1.32 \pm 0.2 \mu \mathrm{M} \mathrm{min}^{-1}$ was observed. However, the efficiency of 4-CB (1 mM) elimination after 24-h incubation reached $40 \%$ in the absence of $\alpha$-tocopherol and $100 \%$ in the presence of the antioxidant (Ponce et al. 2011).

In our experiments, butyltins were identified separately in the fungal biomass and the supernatant (data not shown). On this basis, the high affinity of DBT with the biomass was discovered. During the first hours of the study, as much as $69 \%$ of the initial concentration of the compound was attached to the $M$. robertsii mycelium. On the other hand, the location of the monobutyl derivative was dependent on the oxygen supply and incubation time. In the fungal cultures conducted in the bioreactor, continuous MBT excretion to the growth medium was detected. However, in the flask cultivation of the fungus, MBT was mostly transported to the substrate only for $24 \mathrm{~h}$. Afterwards, accumulation of the monobutyl compound was observed in the hyphae.
Fig. 3 Growth curve, glucose assimilation, and butyltin utilization by the examined fungus incubated for $72 \mathrm{~h}$ in a bioreactor on synthetic medium supplemented with vitamin C (a) or vitamin $\mathrm{E}(\mathbf{b})$
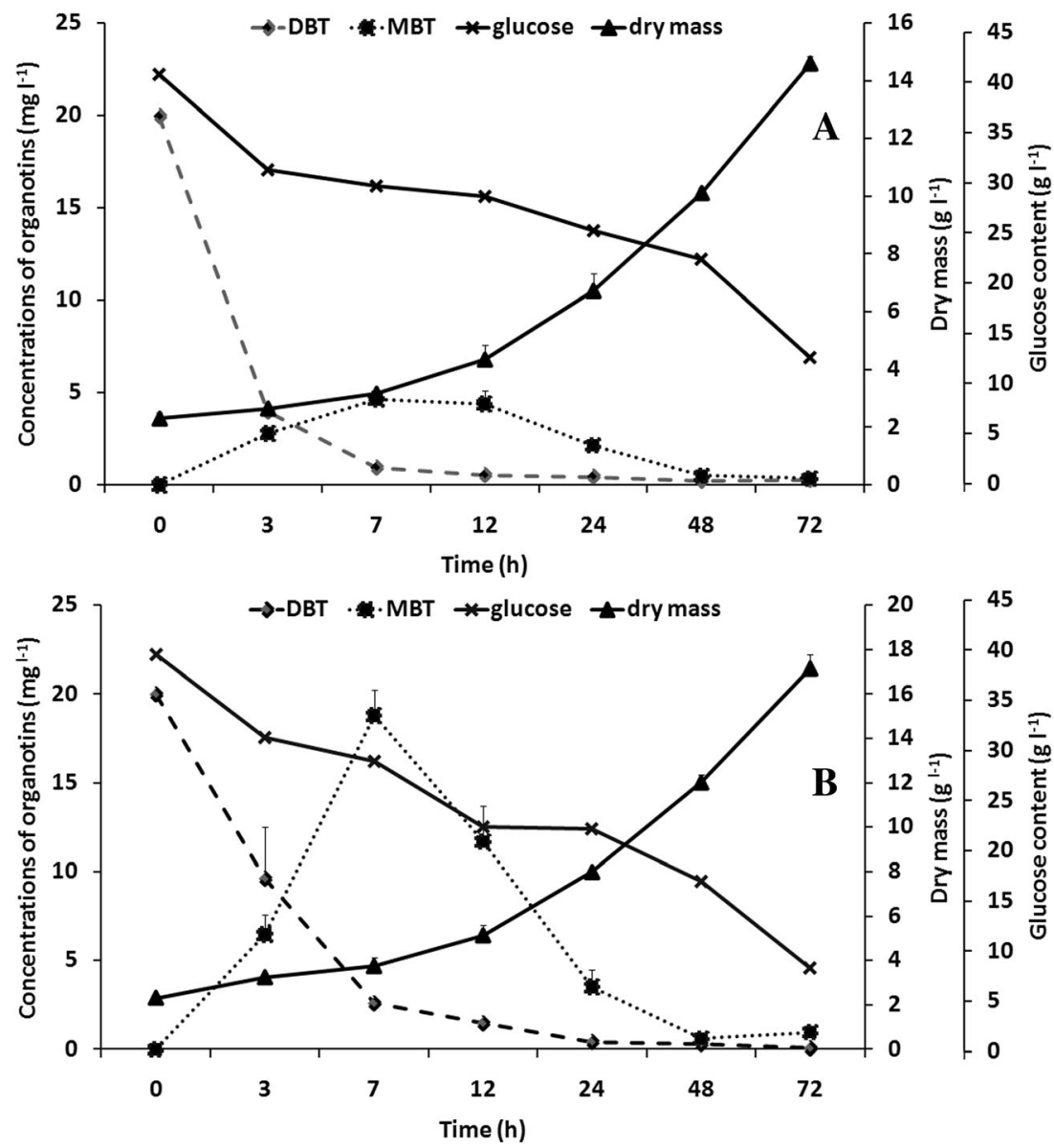
Table 1 Kinetic parameters of $M$. robertsii growth and butyltin removal by the fungus cultivated on flask and bioreactor scale

\begin{tabular}{|c|c|c|c|c|c|c|c|c|c|}
\hline \multirow[t]{2}{*}{ Scale } & \multirow[t]{2}{*}{ Culture } & \multicolumn{3}{|c|}{ Biomass parameters } & \multicolumn{5}{|c|}{ Butyltin degradation parameters } \\
\hline & & $X_{\max }\left(\mathrm{g}^{-1}\right)$ & $\mu_{\max }\left(\mathrm{h}^{-1}\right)$ & $R^{2}$ & $\begin{array}{l}\text { Max DBT } \\
\text { removal }(\%)\end{array}$ & $R^{2}$ & $\begin{array}{l}\text { Max MBT } \\
\text { concentration }\left(\mathrm{mg} \mathrm{l}^{-1}\right)\end{array}$ & $\begin{array}{l}\text { Max MBT } \\
\text { removal }(\%)\end{array}$ & $R^{2}$ \\
\hline \multirow[t]{4}{*}{ Flasks } & Control & $15.75 \pm 0.22$ & 0.060 & 0.997 & - & - & - & - & - \\
\hline & DBT & $19.20 \pm 0.15$ & 0.061 & 1.000 & $98.80 \pm 0.15$ & 0.997 & $10.57 \pm 0.77$ & $77.20 \pm 2.10$ & 0.987 \\
\hline & $\mathrm{DBT}+$ vitamin $\mathrm{C}$ & $21.18 \pm 0.87$ & 0.064 & 0.999 & $94.65 \pm 0.16$ & 0.996 & $16.00 \pm 0.17$ & $67.25 \pm 2.65$ & 0.995 \\
\hline & $\mathrm{DBT}+$ vitamin $\mathrm{E}$ & $22.00 \pm 0.98$ & 0.069 & 0.999 & $96.75 \pm 0.21$ & 0.986 & $11.07 \pm 0.50$ & $69.56 \pm 2.17$ & 0.989 \\
\hline \multirow[t]{4}{*}{ Bioreactor } & Control & $11.07 \pm 0.59$ & 0.078 & 0.988 & - & - & - & - & - \\
\hline & DBT & $10.83 \pm 2.30$ & 0.086 & 0.995 & $98.60 \pm 0.10$ & 0.999 & $13.20 \pm 1.08$ & $95.90 \pm 0.90$ & 0.984 \\
\hline & $\mathrm{DBT}+$ vitamin $\mathrm{C}$ & $14.58 \pm 0.28$ & 0.063 & 1.000 & $98.60 \pm 0.05$ & 0.998 & $4.64 \pm 0.20$ & $92.46 \pm 0.22$ & 0.993 \\
\hline & $\mathrm{DBT}+$ vitamin $\mathrm{E}$ & $17.15 \pm 0.64$ & 0.063 & 0.999 & $99.65 \pm 0.20$ & 0.996 & $18.78 \pm 1.43$ & $94.94 \pm 0.05$ & 0.877 \\
\hline
\end{tabular}

$R^{2}$ coefficients refer to polynomial regression $(n=4)$

A summary of the most important kinetic parameters of the $M$. robertsii growth and its efficiency in butyltin biodegradation is presented in Table 1, i.e., maximum values of biomass $\left(X_{\max }\right)$, specific growth rate $\left(\mu_{\max }\right)$, DBT removal $(\%)$, MBT concentrations, and MBT removal (\%). The regression coefficients $\left(R^{2}\right)$ were higher than 0.98 in all cases, except for MBT removal (\%) in the batch culture of the $M$. robertsii supplemented with DBT and vitamin E, where $R^{2}$ was equal to 0.877 . The values of $R^{2}$ suggested at least good or very good fitting of the model, which confirmed its suitability for use in biodegradation processes.

Intensive aeration and the presence of vitamin $\mathrm{C}$ and glucose in medium ensured optimal conditions for fungal growth and butyltin biodegradation. The use of the nutrients is not cost-effective. However, the application of agricultural wastes as a rich source of carbon and energy for microorganisms (Singh and Nain 2014) is the first step in the reduction of costs with a simultaneous enhancement of biodegradation efficiency. Cometabolism could be a novel way of facilitating the removal of not only butyltins but also other pollutants.

\section{A qualitative analysis of butyltin metabolites}

The extracts of the $M$. robertsii cultures from the exponential growth phase were chosen for the studies because of the rapid decrease in the DBT level at that time. The metabolites formed during the organotin biotransformation were qualitatively analyzed using gas and liquid chromatography coupled with mass spectrometry.

MBT, the primary by-product of DBT biotransformation by the $M$. robertsii cells, was determined with GC-MS/MS. HPLC-MS/MS chromatograms revealed the presence of DBT and MBT and the formation of its metabolite at retention time 4.15 (Fig. 4). The retention times for DBT and MBT were 4.0 and 4.6, respectively. The spectrum of the analyte showed ions 197 and 179, and the appearance of the $\left[\mathrm{SnH}_{3}\right]^{+}$ion at $\mathrm{m} / z, 123$
Fig. 4 Mass spectrum showing the main ion group of $\mathrm{OHBuSnH}_{2}$ acquired on the first day of the $M$. robertsii incubation in the presence of DBT

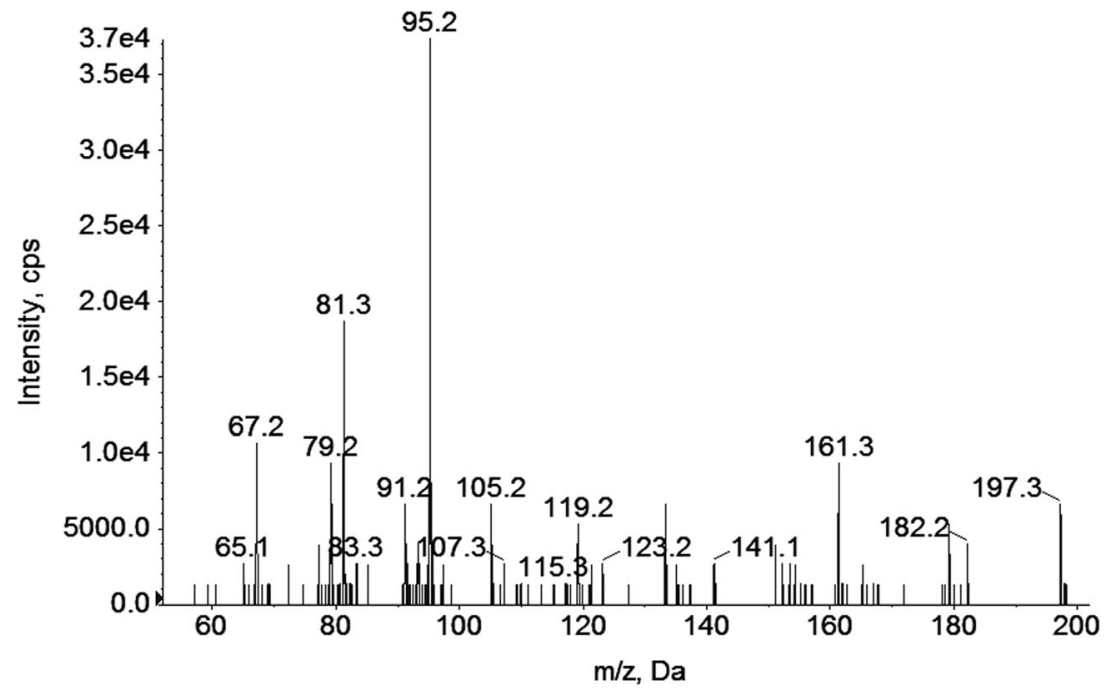




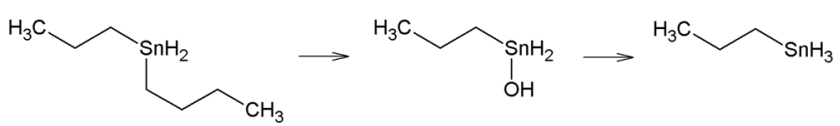

Fig. 5 A proposed pathway of DBT biodegradation by the $M$. robertsii strain

and all of these exhibited a typical tin isotopic pattern. The difference in masses at 18 Da between 197 and 179 $\left[\mathrm{BuSnH}_{2}\right]^{+}$indicated that the obtained compound could be the hydroxylated derivative of MBT- $\mathrm{OHBuSnH}_{2}$. The hydroxylated derivative of DBT was determined in samples from the first to the third day of the $M$. robertsii incubation. Therefore, considering the obtained results, it seems that the presence of $\mathrm{OHBuSnH}_{2}$ was probably associated with DBT debutylation by the fungal strain (Fig. 5). A similar mechanism was described by Suzuki et al. (1992) and Matsuda et al. (1993) for TBT or DBT, which were metabolized either in vitro or in vivo by rat or fish liver microsome enzyme systems to MBT, DBT, and/or hydroxylated products at the third and fourth positions of DBT or TBT.

\section{Fungal cell morphology}

Based on macroscopic observations during the experiments, the morphological modifications of the $M$. robertsii pellets (from the exponential growth phase) cultivated under various levels of oxygen supply were noted (Supplementary Fig. 1). Primarily, the shape and size of fungal morphological forms were examined. In the conditions of intensified oxygenation, the fungus grew as a non-spherical, dispersed form with long hyphae, while the fungus cultivated in flasks formed spherical, densely packed pellets with single hyphae outside the peripheral region. According to Papagianni's (2004) hypothesis, hyphae aggregation and growth as pellets occur as a result of insufficient oxygen levels in the growth medium.

In order to characterize the $M$. robertsii cell morphology, the ratio between the pellet core and the projected area of the whole pellet was calculated (Table 2). A high ratio implied that the pellet had a "smooth" morphology while lower values of the ratio suggested a hairy morphology (Różalska et al. 2014). Densely packed fungal pellets with a smooth morphology were noted in the flask cultures, unlike the pellets from batch cultivation which had a large, actively growing hairy zone. Due to the exposure to DBT and supplementation with vitamins $\mathrm{C}$ or $\mathrm{E}$, an increase in the hairy zone was observed. Moreover, there was a positive correlation $(r=0.803)$ between the degree of hyphae compaction and the efficiency of DBT biodegradation. Papagianni (2004) also reported that hyphae surrounding the pellet core are characterized by high activity. Taking into account the results obtained for the M. robertsii during butyltin and 4-n-nonylphenol biotransformation (Różalska et al. 2014), hyphae compaction seems to be a common feature of the xenobiotic metabolism by fungi from the genus Metarhizium.

\section{Analysis of lipid peroxidation products}

Dibutyltin contributes indirectly to lipid oxidation by inducing reactive oxygen species (Chantong et al. 2014). MDA is the most mutagenic among secondary products formed during the process of lipid peroxidation (Ayala et al. 2014). In this study, quantitative analyses of MDA, one of the most popular and reliable markers of oxidative stress, were conducted with the use of liquid chromatography coupled with mass spectrometry. Extracts of the M. robertsii batch cultures exposed to DBT (with or without the antioxidants) from the exponential growth phase were examined. The highest level of MDA, i.e., $151 \mu \mathrm{M} \mathrm{l}^{-1}$, was determined for those fungal cells supplemented with the organotin alone. In the presence of ascorbic acid and $\alpha$-tocopherol, a decrease in the MDA amount was observed, about 45 and $2 \%$, respectively. The same effect was characterized by Lu et al. (2007), who investigated pancreatic damage in rats induced by the presence of dibutyltin dichloride. The action of AA, determined as soothing, was supported by the measurement of reduced MDA levels after the antioxidant treatment ( $\mathrm{Lu}$ et al. 2007). In order to detect MDA in the organs of rabbits exposed to stannous chloride (the ROS inducer), a reaction with thiobarbituric acid was applied. Researchers have reported that treatment with ascorbic acid causes a decrease in the TBARS levels in all tested organs (El-Demerdash et al. 2005). These findings are consistent with our results, despite the use of different test organisms.

Undoubtedly, the reduction of MDA quantity in fungal membranes damaged by DBT was a result of the efficient process of free radical scavenging by ascorbic acid. Consequently, restriction of oxidative stress was the main

Table 2 The ratio between the pellet core and the projected area of the whole pellet of the $M$. robertsii strain cultivated in flasks and in a bioreactor on synthetic medium in the absence of DBT, in the presence of DBT alone, or in a mixture with one of the vitamins

\begin{tabular}{lllll}
\hline & Control & DBT & DBT + vitamin C & DBT + vitamin E \\
\hline Cultures in flasks & $0.732 \pm 0.076$ & $0.950 \pm 0.029$ & n.t. & n.t. \\
Cultures in bioreactor & $0.430 \pm 0.020$ & $0.562 \pm 0.006$ & $0.629 \pm 0.048$ & $0.536 \pm 0.090$ \\
\hline
\end{tabular}

n.t. not tested 
reason for the improved yield of the process of DBT biodegradation by the examined fungus.

\section{Conclusions}

This report is the first to show that the $M$. robertsii can degrade both DBT and MBT with high levels of efficiency. An additional supply of oxygen led to a hairy morphology for the $M$. robertsii hyphae instead of densely interwoven pellets. A facilitated exchange of nutrients and respiratory gasses between the biomass and growth medium contributed to intensive fungal growth and finally improved butyltin biodegradation. Due to simultaneous utilization of glucose and the organotins, the cometabolic character of the described process is suggested. Moreover, supplementation of the growth medium with ascorbic acid protects fungal cells exposed to DBT from oxidative stress.

Acknowledgements This study was supported by the National Science Centre, Poland (Project No. 2015/19/N/NZ9/00459).

Open Access This article is distributed under the terms of the Creative Commons Attribution 4.0 International License (http:// creativecommons.org/licenses/by/4.0/), which permits unrestricted use, distribution, and reproduction in any medium, provided you give appropriate credit to the original author(s) and the source, provide a link to the Creative Commons license, and indicate if changes were made.

\section{References}

Albalat A, Potrykus J, Pempkowiak J, Porte C (2002) Assessment of organotin pollution along the Polish coast (Baltic Sea) by using mussels and fish as sentinel organisms. Chemosphere 47:165-171

Ayala A, Muñoz MF, Argüelles A (2014) Lipid peroxidation: production, metabolism, and signaling mechanisms of malondialdehyde and 4hydroxy-2-nonenal. Oxidative Med Cell Longev. doi:10.1155/2014/ 360438

Bernat P, Długoński J (2006) Acceleration of tributyltin chloride (TBT) degradation in liquid cultures of the filamentous fungus Cunninghamella elegans. Chemosphere 62(1):3-8

Bernat P, Długoński J (2009) Isolation of Streptomyces sp. strain capable of butyltin compounds degradation with high efficiency. J Hazard Mater 171:660-664

Bernat P, Szewczyk R, Krupiński M, Długoński J (2013) Butyltins degradation by Cunninghamella elegans and Cochliobolus lunatus coculture. J Hazard Mater 246-247:277-282

Bernat P, Siewiera P, Soboń A, Długoński J (2014) Phospholipids and protein adaptation of Pseudomonas sp. to the xenoestrogen tributyltin chloride (TBT). World J Microbiol Biotechnol 30:2343-2350

Bilodeau K, Couet F, Boccafoschi F, Mantovani D (2005) Design of a perfusion bioreactor specific to the regeneration of vascular tissues under mechanical stresses. Artif Organs 29:906-912

Boswell CD, Nienow AW, Gill NK, Kocharunchitt S, Hewitt CJ (2003) The impact of fluid mechanical stress on Saccharomyces cerevisiae cells during continuous cultivation in an agitated, aerated bioreactor; its implication for mixing in the brewing process and aerobic fermentations. Food Bioprod Process 81:23-32
Bouchard N, Pelletier É, Fournier M (1999) Effects of butyltin compounds on phagocytic activity of hemocytes from three marine bivalves. Environ Toxicol Chem 18(3):519-522

Casas López JL, Sánchez Pérez JA, Fernández Sevilla JM, Rodríguez Porcel EM, Chisti Y (2005) Pellet morphology, culture rheology and lovastatin production in cultures of Aspergillus terreus. J Biotechnol 116(1):61-77

Chamsartra S, Hewitt CJ, Nienow AW (2005) The impact of fluid mechanical stress on Corynebacterium glutamicum during continuous cultivation in an agitated bioreactor. Biotechnol Lett 27:693-700

Chantong B, Kratschmar DV, Lister A, Odermatt A (2014) Dibutyltin promotes oxidative stress and increases inflammatory mediators in BV-2 microglia cells. Toxicol Lett 230(2):177-187

Chen CW, Chen CF, Ju YR, Dong CD (2016) Assessment of the bioaccumulation and biodegradation of butyltin compounds by Thalamita crenata in Kaohsiung Harbor, Taiwan. Int Biodeterior Biodegradation 113:97-104

Csallany AS, Guan MD, Manwaring JD, Addis PB (1984) Free malonaldehyde determination in tissues by high-performance liquid chromatography. Anal Biochem 142:277-283

El-Demerdash FM, Yousef MI, Zoheir MA (2005) Stannous chloride induces alterations in enzyme activities, lipid peroxidation and histopathology in male rabbit: antioxidant role of vitamin C. Food Chem Toxicol 43(12):1743-1752

Frouin H, Lebeuf M, Saint-Louis R, Hammill M, Pelletier E, Fournier M (2008) Toxic effects of tributyltin and its metabolites on harbour seal (Phocavitulina) immune cells in vitro. Aquat Toxicol 90(3):243251

Garcia-Ochoa F, Gomez E (2009) Bioreactor scale-up and oxygen transfer rate in microbial processes: an overview. Biotechnol Adv 27: 153-176

Jenkins SM, Ehman K, Barone S Jr (2004) Structure-activity comparison of organotin species: dibutyltin is a developmental neurotoxicant in vitro and in vivo. Behav Brain Res 151:1-12

Lee JS, Little BJ (2015) Technical note: electrochemical and chemical complications resulting from yeast extract addition to stimulate microbial growth. Corrosion 71(12):1434-1440

Li Y, Schellhorn HE (2007) New developments and novel therapeutic perspectives for vitamin C. J Nutr 137(10):2171-2184

Lobos JH, Leib TK, Su TM (1992) Biodegradation of bisphenol A and other bisphenols by a gram-negative aerobic bacterium. Appl Environ Microbiol 58:1823-1831

Lu XL, Song YH, Fu YB, Si JM, Qian KD (2007) Ascorbic acid alleviates pancreatic damage induced by dibutyltin dichloride (DBTC) in rats. Yonsei Med J 48(6):1028-1034

Matsuda R, Suzuki T, Saito Y (1993) Metabolism of tri-n-butyltin chloride in male rats. J Agric Food Chem 41:489-495

Moscoso F, Teijiz I, Deive FJ, Sanromán MA (2012) Efficient PAHs biodegradation by a bacterial consortium at flask and bioreactor scale. Bioresour Technol 119:270-276

Moscoso F, Deive FJ, Longo MA, Sanromán MA (2015) Insights into polyaromatic hydrocarbon biodegradation by Pseudomonas stutzeri CECT 930: operation at bioreactor scale and metabolic pathways. Int J Environ Sci Technol 12:1243-1252

Moser VC, McGee JK, Ehman KD (2009) Concentration and persistence of tin in rat brain and blood following dibutyltin exposure during development. J Toxicol Environ Health A 72(1):47-52

Nemanič TM, Milačič R, Ščančar J (2009) A survey of organotin compounds in the Northern Adriatic Sea. Water Air Soil Pollut 196:211224

Nesci S, Ventrella V, Trombetti F, Pirini M, Borgatti AR, Pagliarani A (2011) Tributyltin (TBT) and dibutyltin (DBT) differently inhibit the mitochondria Mg-ATPase activity in mussel digestive gland. Toxicol in Vitro 25:117-124

Nielsen JB, Strand J (2002) Butyltin compounds in human liver. Environ Res 88:129-133 
Papagianni M (2004) Fungal morphology and metabolite production in submerged mycelial processes. Biotechnol Adv 22(3):189-259

Ponce BL, Latorre VK, González M, Seeger M (2011) Antioxidant compounds improved PCB-degradation by Burkholderia xenovorans strain LB400. Enzym Microb Technol 49(6-7):509-516

Różalska S, Glińska S, Długoński J (2014) Metarhizium robertsii morphological flexibility during nonylphenol removal. Int Biodeterior Biodegradation 95:285-293

Sakultantimetha A, Keenan HE, Beattie TK, Bangkedphol S, Cavoura O (2011) Bioremediation of tributyltin contaminated sediment: degradation enhancement and improvement of bioavailability to promote treatment processes. Chemosphere 83:680-686

Siewiera P, Bernat P, Różalska S, Długoński J (2015) Estradiol improves tributyltin degradation by the filamentous fungus Metarhizium robertsii. Int Biodeterior Biodegradation 104:258-263

Singh S, Nain L (2014) Microorganisms in the conversion of agricultural wastes to compost. Proc Indian Natn Sci Acad 80:473-481

Słaba M, Gajewska E, Bernat P, Fornalska M, Długoński J (2013) Adaptive alterations in the fatty acids composition under induced oxidative stress in heavy metal-tolerant filamentous fungus Paecilomyces marquandii cultured in ascorbic acid presence. Environ Sci Pollut R 20:3423-3434
Soboń A, Szewczyk R, Długoński J (2016) Tributyltin (TBT) biodegradation induces oxidative stress of Cunninghamella echinulata. Int Biodeterior Biodegradation 107:92-101

Sontag TJ, Parker RS (2002) Cytochrome P450 $\omega$-hydroxylase pathway of tocopherol catabolism. Novel mechanism of regulation of vitamin E status. J Biol Chem 277(28):25290-25296

Suzuki T, Matsuda R, Saito Y (1992) Molecular species of tri-n-butyltin compounds in marine products. J Agric Food Chem 40(8):14371443

Tsang CK, Lau PS, Tam NFY, Wong YS (1999) Biodegradation capacity of tributyltin by two Chlorella species. Environ Pollut 105:289-297

Wei R, Li G, Seymour AB (2010) High-throughput and multiplexed LC/MS/ MRM method for targeted metabolomics. Anal Chem 82:5527-5533

Whalen MM, Loganathan BG, Kannan K (1999) Immunotoxicity of environmentally relevant concentrations of butyltins on human natural killer cells in vitro. Environ Res 81:108-116

White JS, Tobin JM, Cooney JJ (1999) Organotin compounds and their interactions with microorganisms. Can J Microbiol 45:541-554

Yáñez J, Riffo P, Santander P, Mansilla HD, Mondaca MA, Campos V, Amarasiriwardena D (2015) Biodegradation of tributyltin (TBT) by extremophile bacteria from Atacama Desert and speciation of tin byproducts. Bull Environ Contam Toxicol 95:126-130 